\title{
Codimension Reduction for Real Submanifolds of a Complex Hyperbolic Space
}

\author{
SHIN-ICHI KAWAMOTO
}

ABSTRACT. We study real submanifolds of a complex hyperbolic space and prove a codimension reduction theorem.

\section{o. INTRODUCTION.}

Recently Okumura ([3]) defined holomorphic first normal space for real submanifolds of a Kaehler manifold and proved a codimension reduction theorem for real submanifolds of a complex projective space. Namely, he showed following:

Theorem. Let $M$ be a connected $n$-dimensional real submanifold of a real $(n+p)$-dimensional complex projective space $\mathbf{C} P^{(n+p) / 2}$ and let $N_{0}(x)$ be the orthogonal complement of first normal space in $T_{x}^{\perp}(M)$. We put $H_{0}(x)=J N_{0}(x) \cap N_{0}(x)$ and let $H(x)$ be a $J$-invariant subspace of $H_{0}(x)$ where $J$ is complex structure of $\mathbf{C} P^{(n+p) / 2}$. If the orthogonal complement $H_{2}(x)$ of $H(x)$ in $T_{x}^{\perp}(M)$ is invariant under parallel translation with respect to the normal connection and if $q$ is the constant

1991 Mathematics Subject Classification: 53B25, 53B30.

Editorial Complutense. Madrid, 1994. 
dimension of $\mathrm{H}_{2}(x)$, then there exists a real $(n+q)$-dimensional totally geodesic complex projective subspace $\mathrm{C} P^{(n+q) / 2}$ in $\mathrm{C} P^{(n+p) / 2}$ such that $M \subset \mathbf{C} P^{(n+q) / 2}$.

The purpose of this paper is to prove that the similar result to the above theorem is still hold in a submanifold of complex hyperbolic space.

The auther would like to express his thanks to Professors M. Okumura and $M$. Kimura for their valuable suggestions.

\section{CODIMENSION REDUCTION FOR SUBMANIFOLDS OF ANTI-DE SITTER SPACE.}

Let $R_{2}^{n+1}$ be a real vector space of $(n+1)$ dimension with a pseudoRiemannian metric $\bar{g}$ of signature $(n-1,2)$ given by

$$
\bar{g}(x, y)=-x_{0} y_{0}-x_{1} y_{1}+\sum_{i=2}^{n} x_{i} y_{i}
$$

where $x={ }^{t}\left(x_{0}, x_{1}, \ldots, x_{n}\right), y={ }^{t}\left(y_{0}, y_{1}, \ldots, y_{n}\right) \in \mathbf{R}^{n+1}$. Let $H_{1}^{n}=$ $\left\{x \in \mathbf{R}_{2}^{n+1} \mid g(x, x)=-1\right\}$. Then the hypersurface $H_{1}^{n}$ is a Lorentzian manifold with the induced Lorentzian metric $\tilde{g}$ of constant sectional curvature -1 . We call it $n$-dimensional anti-De Sitter space.

Let $H_{1}^{n+p}$ be an $(n+p)$-dimensional anti-De Sitter space and let $i: M \rightarrow H_{1}^{n+p}$ be an isometric immersion of a connected $n$-dimensional Lorentzian manifold with the Lorentzian metric $g$ into $H_{1}^{n+p}$. Then the tangent bundle $T(M)$ is identified with a subbundle of $T\left(H_{1}^{n+p}\right)$ and the normal bundle $T^{\perp}(M)$ is a subbundle of $T\left(H_{1}^{n+p}\right)$ consisting of all element in $T\left(H_{1}^{n+p}\right)$ which are orthogonal to $T(M)$ with respect to $\tilde{g}$. We denote by $\nabla$ and $\tilde{\nabla}$ the Levi-Civita connection of $M$ and $H_{1}^{n+p}$ respectively and $D$ the induced normal connection from $\tilde{\nabla}$ to $T^{\perp}(M)$. Then they are related by the following Gauss and Weingarten formulae:

$$
\begin{gathered}
\tilde{\nabla}_{i X} i Y=i \nabla X Y+h(X, Y) \\
\tilde{\nabla}_{i X} \xi=-i A_{\xi} X+D_{X} \xi
\end{gathered}
$$


where $\xi \in T^{\perp}(M), h(X, Y)$ is the second fundamental form and $A_{\xi}$ is a symmetric linear transformation of $T(M)$ which is called the shape operator with respect to $\xi$. They satisfy

$$
\tilde{g}(h(X, Y), \xi)=g\left(A_{\xi} X, Y\right) .
$$

Next let $N_{0}(x)=\left\{\xi \in T_{x}^{\perp}(M) \mid A_{\xi}=0\right\}$. The first normal space $N_{1}(x)$ is defined to be the orthogonal complement of $N_{0}(x)$ in $T_{x}^{\perp}(M)$.

Theorem 1.1. Let $i: M \rightarrow H_{1}^{n+p}$ be as above. Let $N_{2}(x)$ be a subspace of $T_{x}^{\perp}(M)$ such that $N_{1}(x) \subset N_{2}(x)$. If $N_{2}(x)$ is invariant under parallel translation with respect to the normal connection and if $q$ is the constant dimension of $N_{2}(x)$, then there exists a totally geodesic anti-De Sitter subspace $H_{1}^{n+q}$ of $H_{1}^{n+p}$ such that $i(M) \subset H^{n+q}$.

Proof. We consider $H_{1}^{n+p}$ as a hypersurface of $\mathbf{R}_{2}^{n+p+1}$. Let $x \in M$ and let $\xi=i \overrightarrow{i(x)}$ be the position vector. Then $\xi(x)$ is normal to $H_{1}^{n+p}$ and $\bar{g}(\xi(x), \xi(x))=-1$ where $\bar{g}$ is the metric of $R_{2}^{n+p+1}$. Let $\bar{\nabla}$ be the Levi-Civita connection on $\mathbf{R}_{2}^{n+p+1}$ with respect to $\bar{g}$ and $\varphi$ be an immersion from $H_{1}^{n+p}$ to $R_{2}^{n+p+1}$. Then

$$
\begin{aligned}
\nabla \varphi X \xi & =\varphi X \\
\bar{\nabla} \varphi X \varphi Y & =\varphi \tilde{\nabla} X Y-\tilde{g}(X, Y) \xi
\end{aligned}
$$

where $X, Y \in T_{x}\left(H_{1}^{n+p}\right)$. For $x \in M$ let $P(x)=T_{x}(M)+N_{2}(x)$. For any $x \in M$ there exist orthonormal normal vector fields $\xi_{1}, \ldots, \xi_{p}$ defined in a neighborhood $U$ of $x$ such that:

(a) For any $y \in U, \xi_{1}(y), \ldots, \xi_{q}(y)$ span $N_{2}(y)$, and $\xi_{q+1}(y), \ldots, \xi_{p}(y)$ span $N(y)$ where $N(x)$ is the orthogonal complement of $N_{2}(x)$ in $T^{\perp}(M)$.

(b) $\tilde{\nabla}_{i X} \xi_{\alpha}=0$ in $U$ for $\alpha \geq q+1$ and $X$ tangent to $M$.

(c) $\{P(y) \mid y \in U\}$ is invariant under parallel translation with respect to the connection $\tilde{\nabla}$ along any curve in $U$ (see [1]). Then $\bar{\nabla}_{\varphi(i X)} \varphi \xi_{\alpha}=\tilde{\nabla}_{i X} \xi_{\alpha}$ for $X$ tangent to $M$. Let $D^{\prime}$ be the normal connection in the normal bundle $T^{\perp}(M)$ of $M$ in $\mathbf{R}_{2}^{n+p+1}$. Then 
$N_{2}(x)+\operatorname{span}\{\xi(x)\}$ is invariant under parallel translation with respect to $D^{\prime}$. Further,

$$
W(x)=T_{x}(M)+N_{2}(x)+\operatorname{span}\{\xi(x)\}
$$

is invariant under parallel translation with respect to $\bar{\nabla}$. Next we shall show that there exists a totally geodesic submanifold $H_{1}^{n+q}$ of $H_{1}^{n+p}$ such that $i(M) \subset H_{1}^{n+q}$. Define functions $f_{\alpha}$ on $U$ by $f_{\alpha}=\bar{g}\left(i \overrightarrow{i(x)}, \varphi \xi_{\alpha}\right)$ for $\alpha \geq q+1$.

$$
\left.\varphi(i X) \cdot f_{\alpha}=\bar{g}\left(\bar{\nabla}_{\varphi(i X)} \overrightarrow{i(x)}, \varphi \xi_{\alpha}\right)+\bar{g}\left(\overrightarrow{i(x)}, \varphi \xi_{\alpha}\right), \bar{\nabla} \varphi(i X) \varphi \xi_{\alpha}\right)=0
$$

Thus $f_{q+1}, \ldots, f_{p}$ are constant. Put

$$
f_{\alpha}=C_{\alpha}(=\text { constant })(\alpha \geq q+1)
$$

And put $\overrightarrow{i(\vec{x})}=\left(x_{0}, \ldots, x_{n+p}\right)$ and $\varphi \xi_{\alpha}=\left(\xi_{\alpha}^{0}, \ldots, \xi_{\alpha}^{n+p}\right)$. Then (1.6) can be written

$$
\left\{\begin{array}{l}
-\xi_{q+1}^{0} x_{0}-\xi_{q+1}^{1} x_{1}+\sum_{i=1}^{n+p} \xi_{q+1}^{i} x_{i}=C_{q+1}, \\
\vdots \\
-\xi_{p}^{0} x_{0}-\xi_{p}^{1} x_{1}+\sum_{i=2}^{n+p} \xi_{p}^{i} x_{i}=C_{p} .
\end{array}\right.
$$

Since $\xi_{q+1}, \ldots, \xi_{p}$ are linearly independent, $U$ lies in the intersection of $p-q$ hyperplanes and the dimension of the hyperplane is $n+q+1$. As the normal vectors of the intersection $W^{\prime}$ are $\xi_{q+1}, \ldots, \xi_{p}$, they span $N(x)$. Since $W^{\prime}$ is affine space, $W^{\prime}$ is the orthogonal complement of $N(x)$ in $T_{x}\left(\mathbf{R}_{2}^{n+p+1}\right)$. On the other hand, the orthogonal complement of $N(x)$ in $T_{x}\left(\mathbf{R}_{2}^{n+p+1}\right)$ is $T_{x}(M)+N_{2}(x)+\operatorname{span}\{\xi(x)\}(=W(x))$. Therefore $W^{\prime}=$ $W$. We may assume that the point $(1,0, \ldots, 0)$ is in $U . W(x)$ contains $\xi$, and if $\xi=(1,0, \ldots, 0)$, then $W(x)$ passes through the origin of $\mathbf{R}_{2}^{n+p+1}$. Thus $W(x)=\mathbf{R}^{n+q+1}$. Moreover since $M$ is Lorentzian submanifold and $\xi$ is the position vector, the signature of the induced metric of 
$\mathbf{R}^{n+q+1}$ is $(n+q-1,2)$. Then $W^{\prime}=\mathbf{R}_{2}^{n+q+1}$. Thus $H_{1}^{n+p} \cap \mathbf{R}_{2}^{n+q+1}$ is totally geodesic $H_{1}^{n+p}$, that is,

$$
i(U) \subset H_{1}^{n+q}=H_{1}^{n+p} \cap \mathbf{R}_{2}^{n+q+1} .
$$

Hence Theorem 1.1. is true locally. In entirely the same way as in [1], we can get the global result. This completes the proof.

\section{REAL SUBMANIFOLDS OF A KAEHLER MANIFOLD AND HOLOMORPHIC FIRST NORMAL SPACE.}

Let $\bar{M}$ be a real $(n+p)$-dimensional Kaehler manifold with Kaehler structure $(J,<,>)$, that is, $J$ is the endomorphism of the tangent bundle $T(\bar{M})$ satisfying $J^{2}=$-identity and $<,>$ the Riemannian metric of $\bar{M}$ satisfying the Hermitian condition $\langle J \bar{X}, J \bar{Y}\rangle=\langle\bar{X}, \bar{Y}\rangle$ for any $\bar{X}, \bar{Y} \in T(\bar{M})$.

Let $M$ be a connected $n$-dimensional submanifold and let $i$ be the isometric immersion. For any $X \in T(M)$ the transform $J i X$ is written as a sum of its tangential parts iFX and the normal parts $u(X)$ in the following way:

$$
J i X=i F X+u(X)
$$

Then $F$ is an endmorphism on the tangent bundle $T(M)$ and $u$ is a normal valued 1 -form on the tangent bundle. In the same way, for any $\xi \in T^{\perp}(M)$, the transform $J \xi$ is written as

$$
J \xi=-i U_{\xi}+P \xi
$$

where $P$ defines an endomorphism on the normal bundle $T^{\perp}(M)$. It is easily verified that

$$
g\left(X, U_{\xi}\right)=\langle u(X), \xi>
$$

where $g$ is the Riemannian metric which is induced from the Riemannian metric $<$, $>$.

We define the holomorphic first normal space. We put $H_{0}(x)=$ $J N_{0}(x) \cap N_{0}(x)$. Then $H_{0}(x)$ is the maximal $J$-invariant subspace of 
$N_{0}(x)$. Since $J$ is isomorphism, we see that $J H_{0}(x)=H_{0}(x)$. Making use of (2.2), we can easily prove the following

Proposition 2.1. ([3]) For any $\xi \in H_{0}(x)$, we have $A_{\xi}=0$ and $U_{\xi}=0$.

Definition ([3]) The holomorphic first normal space $H_{1}(x)$ is the orthogonal complement of $H_{0}(x)$ in $T_{x}^{\perp}(M)$.

Proposition 2.2. ([3]) If $M$ is a complex submanifold of a Kaehler manifold, then $H_{1}(x)=N_{1}(x)$.

Proposition 2.3. ([3]) Let $H(x)$ be a $J$-invariant subspace of $H_{0}(x)$ and let $H_{2}(x)$ be the orthogonal complement of $H(x)$ in $T_{x}^{\perp}(M)$. Then $T_{x}(M)+H_{2}(x)$ is a $J$-invariant subspace of $T_{x}(\bar{M})$.

\section{CODIMENSION REDUCTION FOR SUBMANIFOLDS OF COMPLEX HYPERBOLIC SPACE.}

In this section, we consider the case that the ambient manifold $\bar{M}$ is a complex hyperbolic space $\mathrm{CH} H^{(n+p) / 2}$ with the Bergmann metric of constant holomorphic sectional curvature -4 . Given a real $n$ dimensional submanifold $M$ of $\mathbf{C} H^{(n+p) / 2}$, one can construct a Lorentzian submanifold $M^{\prime}$ with time like totally geodesic fibres and projection $\pi^{\prime}: M^{\prime} \rightarrow M$ such that the diagram ([2])

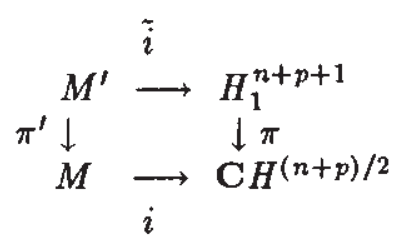

is commutative ( $\tilde{i}$ being the isometric immersion). Let $V^{\prime}$ be the unit vector field tangent to the fibre of $M^{\prime}$. Then $\tilde{i} V^{\prime}$ is the unit vector field tangent to the fibre of $H_{1}^{n+p+1}$. We denote by $g^{\prime}$ and $\nabla^{\prime}$ the Lorentzian metric and the Levi-Civita connection of $M^{\prime}$ respectively. Also we denote by $F$ and $X^{*}$ the fundamental tensor of the submersion 
$\pi^{\prime}$ and the horizontal lift for $X \in T(M)$ respectively. In the same way, $\xi^{*}$ is the horizontal lift of the normal field $\xi \in T^{\perp}(M)$. The fundamental equations for the submersion $\pi^{\prime}$ are given as following ([4]):

$$
\begin{aligned}
\nabla_{X}^{\prime} * Y^{*} & =(\nabla X Y)^{*}+g^{\prime}\left((F X)^{*}, Y^{*}\right) V^{\prime} \\
\nabla_{X}^{\prime} * V^{\prime} & =\nabla_{V^{\prime}}^{\prime} X^{*}=(F X)^{*}
\end{aligned}
$$

where $\nabla$ is the Levi-Civita connection of $M$. The similar equations are valid for the submersion $\pi: H_{1}^{n+p+1} \rightarrow \mathbf{C} H^{(n+p) / 2}$ when we replace $F$ and $V^{\prime}$ with $J$ and $\tilde{i} V^{\prime}$ respectively. Let $\tilde{g}, \tilde{\nabla}, A^{\prime}$ and $D^{\prime}$ be respectively the Lorentzian metric of $H_{1}^{n+p+1}$, the Levi-Civita connection for $\tilde{g}$, the shape operator and the normal connection of $M^{\prime}$, and let $A$ and $D$ be the shape operator and the normal connection of $M$ respectively. Then ([3]) we have

$$
\begin{aligned}
A_{\xi}^{\prime} * X^{*} & =\left(A_{\xi} * X\right)^{*}-g\left(U_{\xi}, X\right)^{*} V^{\prime} \\
D_{X}^{\prime} * \xi^{\prime} & =\left(D_{X} \xi\right)^{*} \\
A_{\xi}^{\prime} * V^{\prime} & =U_{\xi^{\prime}}^{*} \\
D_{V^{\prime}}^{\prime} \xi^{*} & =(P \xi)^{*}
\end{aligned}
$$

In fact, from the commutativity of the diagram, (2.3) and (3.1) imply

$$
\begin{aligned}
\tilde{\nabla}_{(i X)} * \xi^{*} & =\left(\tilde{\nabla}_{i X} \xi\right)^{*}+g\left((J i X)^{*}, \xi^{*}\right) \tilde{i} V^{\prime} \\
& =-\left(i A_{\xi} X\right)^{*}+\left(D_{X} \xi\right)^{*}+\langle J i X, \xi\rangle^{*} \tilde{i} V^{\prime} \\
& =-\tilde{i}\left(A_{\xi} X\right)^{*}+\langle u(X), \xi\rangle^{*} \tilde{i} V^{\prime}+\left(D_{X} \xi\right)^{*} \\
& =-\tilde{i}\left\{\left(A_{\xi} X\right)^{*}-g\left(U_{\xi}, X\right)^{*} V^{\prime}\right\}+\left(D_{X} \xi\right)^{*} .
\end{aligned}
$$

On the other hand, by the Weingarten formula, we get

$$
\tilde{\nabla}(i X) * \xi^{*}=-\tilde{i} A_{\xi} * X^{*}+D_{X}^{\prime} * \xi^{*}
$$

Comparing (3.7) and (3.8), we have (3.3) and (3.4). 
Lemma 3.1. ([3]) For a point $x^{\prime}$ such that $\pi\left(x^{\prime}\right)=x$, we have $N_{0}^{\prime}\left(x^{\prime}\right)=\left\{\xi^{*} \mid A_{\xi}=0, U_{\xi}=0\right\}$.

Theorem 3.2. Let $i: M \rightarrow \mathbf{C} H^{(n+p) / 2}$ be an isometric immersion of a connected $n$-dimensional real submanifold into a real $(n+p)$ dimensional complex hyperbolic space $\mathrm{CH}^{(n+p) / 2}$ and let $H(x)$ be a $J$ invariant subspace of $H_{0}(x)$. If the orthogonal complement $\mathrm{H}_{2}(x)$ of $H(x)$ in $T_{x}^{\perp}(M)$ is invariant under parallel translation with respect to the normal connection and if the dimension $q$ of $H_{2}(x)$ is constant, then there exists a real $(n+q)$-dimensional totally geodesic complex hyperbolic subspace $\mathbf{C} H^{(n+q) / 2}$ in $\mathbf{C} H^{(n+p) / 2}$ such that $i(M) \subset \mathbf{C} H^{(n+q) / 2}$.

Proof. We construct the principal circle bundle $M^{\prime}$ over $M$ with time like totally geodesic fibre $S^{1}$. We shall show that $H_{2}(x)^{*}$ is invariant under parallel translation with respect to the normal connection. Assume $\xi \in H(x)$. Then $\xi \in H_{0}(x)$ and by Proposition 2.1., we have

$$
A_{\xi}=0 \text { and } U_{\xi}=0 .
$$

From Lemma 3.1., this yields

$$
A_{\xi^{*}}^{\prime}=0
$$

This shows that, for a point $x^{\prime}$ such that $\pi\left(x^{\prime}\right)=x, H(x)^{*}=$ $\left\{\xi^{*} \mid \xi^{*} \in H(x)\right\}$ is a subspace of $N_{0}^{\prime}\left(x^{\prime}\right)$. Hence, the orthogonal complement $H_{2}(x)^{*}=\left\{\xi^{*} \mid \xi \in H_{2}(x)\right\}$ of $H(x)^{*}$ in $T_{x^{\prime}}^{\perp}\left(M^{\prime}\right)$ is a subspace of $T_{x^{\prime}}^{\perp}\left(M^{\prime}\right)$ such that $N_{1}^{\prime}\left(x^{\prime}\right) \subset H_{2}(x)^{*}$. Since $H_{2}(x)$ is invariant under parallel translation with repect to the normal connection, so is $H(x)$, that is, for $\xi \in H(x), D_{X} \xi \in H(x)$, hence, from (3.4) and (3.5), we have $D_{X}^{\prime} * \xi^{*}=\left(D_{X} \xi\right)^{*} \in H(x)^{*}$ and $D_{V^{\prime}}^{\prime} \xi^{*}=(P \xi)^{*} \in H(x)^{*}$. Since $H(x)^{*}$ is invariant under translation with respect to the normal connection of $M^{\prime}$, so is $H_{2}(x)^{*}$. Here from Theorem 1.1., there exists a totally geodesic submanifold $H_{1}^{n+q+1}$ such that $\tilde{i}\left(M^{\prime}\right) \subset H_{1}^{n+q+1}$. Let $U\left(x^{\prime}\right)$ be a neighborhood of $x^{\prime}$ which satisfies $\pi\left(x^{\prime}\right)=x$. For $y^{\prime} \in U\left(x^{\prime}\right)$ with $y=\pi^{\prime}\left(y^{\prime}\right)$, we get

$$
\begin{aligned}
T_{y^{\prime}}\left(H_{1}^{n+q+1}\right) & =T_{y^{\prime}}\left(M^{\prime}\right)+H_{2}(y)^{*} \\
& =\left(T_{y}(M)+H_{2}(y)\right)^{*}+\operatorname{span}\left\{V^{\prime}\right\}
\end{aligned}
$$


The integral curve $S^{1}$ of $\tilde{i} V$ is time like totally geodesic fibre in $H_{1}^{n+q+1}$. Since $H_{1}^{n+q+1}$ is totally geodesic in $H_{1}^{n+p+1}$, the integral curve $S^{1}$ is a geodesic of $H_{1}^{n+q+1}$. We denote by $\mathrm{C} H^{(n+q) / 2}$ the quotient space $H_{1}^{n+q+1} / S^{1}$. Then the Hopf fibration $H_{1}^{n+q+1} \rightarrow \mathrm{CH} H^{(n+q) / 2}$ by the geodesic $S^{1}$ is compatible with the Hopf fibration $\pi: H_{1}^{n+p+1} \rightarrow$ $\mathrm{C} H^{(n+p) / 2}$ and since $H_{1}^{n+q+1}$ is totally geodesic in $H_{1}^{n+p+1}, \mathrm{CH}^{(n+q) / 2}$ is totally geodesic in $\mathbf{C H} H^{(n+p) / 2}$. Hence the diagram

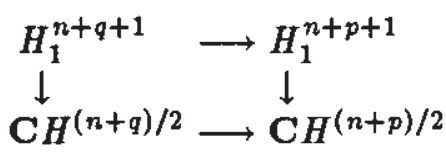

is commutative. Since the tangent space of the $\mathbf{C} H^{(n+q) / 2}$ at $x$ is $T_{x}(M)+H_{2}(x)$, by Proposition 2.3., $\mathrm{CH}^{(\mathrm{n}+q) / 2}$ is $J$-invariant subspace of $\mathrm{CH}^{(n+p) / 2}$ : This completes the proof.

For a complex submanifold $M$, from Proposition 2.2. and Theorem 3.2 ., we have

Corollary. Let $M$ be an n/2-dimensional complex stubmanifold of $\mathrm{CH} H^{(n+p) / 2}$. Suppose a $J$-invariant subspace of the first normal space $N_{1}(x)$ has constant dimension $q$ and $N_{1}(x)$ is parallel with respect to the normal connection. Then there exists a totally geodesic $(n+q)$ dimensional complex hyperbolic subspace $\mathbf{C} H^{(n+q) / 2}$ such that $M \subset$ $\mathrm{C} H^{(n+q) / 2}$.

\section{References.}

[1] Erbacher, J., Reduction of the codimension of an isometric immersion, J. Differential Geometry. 5 (1971), 333-340.

[2] Montiel, S., Real hypersurfaces of a complex hyperboric space, J. Math. Soc. Japan. 37 (1985), 515-535.

[3] Okumura, M., Codimension reduction problem for real submanifold of complex projective space, to appear in Colloquia Mathematica Societatis Janos Bolyai. 56 (1991). 
[4] O'Neill, B. The fundamental equations of a submersion, Michigan Math. J. 13 (1966), 459-469.

Department of Mathematics,

Recibido: 15 de octubre de 1992

Faculty of Science,

Saitama University, Urawa,

Saitaına 338, Japan. 